\title{
Correction: circKDM4C suppresses tumor progression and attenuates doxorubicin resistance by regulating miR-548p/PBLD axis in breast cancer
}

\section{Yiran Liang • Xiaojin Song - Yaming Li • Peng Su - Dianwen Han - Tingting Ma $\cdot$ Renbo Guo $\cdot$ Bing Chen • Wenjing Zhao $\cdot$ Yuting Sang $\cdot$ Ning Zhang Xiaoyan Li $\cdot$ Hanwen Zhang $\cdot$ Ying Liu $\cdot$ Yi Duan $\cdot$ Lijuan Wang Qifeng Yang (1)}

Published online: 25 March 2021

(c) The Author(s), under exclusive licence to Springer Nature Limited 2021

\section{Correction to: Oncogene}

https://doi.org/10.1038/s41388-019-0926-z

Unfortunately, an error occurred in Figs. 61 and S2d. The corrected figures are given below.
Figure S2. Overexpression of circKDM4C inhibits proliferation, migration, and invasion of breast cancer cells. d Cell migration and invasion abilities of breast cancer cells transfected with control or circKDM4C overexpression vectors were evaluated by transwell assay.

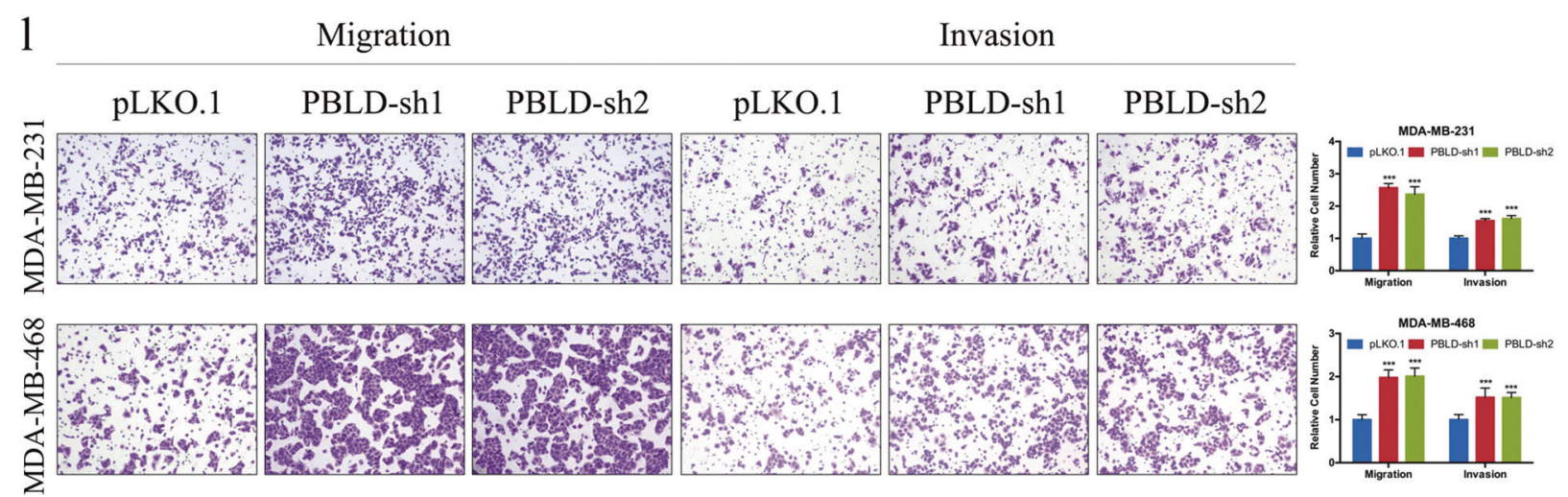

Fig. 6 CircKDM4C inhibited breast cancer cell progression and doxorubicin resistance through protecting PBLD from miR-548p-induced degradation. I Transwell assays revealed that PBLD knockdown promoted migration and invasion abilities of breast cancer cells $(* * P<0.01$; $* * * P<0.001)$ 